\title{
Polynomial Approximations to Integral Transforms
}

\section{By Jet Wimp}

1. Introduction. The symmetric Jacobi polynomials $P_{n}^{(\alpha, \alpha)}(x)$, orthogonal on the interval $-1 \leqq x \leqq 1$, are widely used for approximating functions, but the integral which defines the coefficients for the expansion of a function $g(x)$ in these polynomials usually is quite difficult to evaluate. The problem is simplified if $g(x)$ is an integral transform of the Fourier or Laplace type, since the kernel of the transform generates a series of the above polynomials. The coefficients in such cases are found to be Hankel transforms, which are widely tabulated.

Examples include Chebyshev polynomial expansions of $1 /(x+a)^{k}, \psi(x+a)$, $\log \Gamma(x+a), C i(x)$ and $\operatorname{Si}(x)$.

2. Formulas When $g(x)$ is a Laplace or Fourier Transform. The symmetric Jacobi polynomials [1, v. 2, p. 168] may be defined by

$$
P_{n}^{(\alpha, \alpha)}(x)=\left(\begin{array}{c}
n+\alpha \\
n
\end{array}\right){ }_{2} F_{1}\left[-n, n+2 \alpha+1 ; \alpha+1 ; \frac{1}{2}-\frac{1}{2} x\right] .
$$

A function $g(x)$ satisfying certain conditions has the expansion

$$
g(x)=\sum_{n=0}^{\infty} A_{n} P_{n}^{(\alpha, \alpha)}(x), \quad-1 \leqq x \leqq 1,
$$

where

$$
A_{n}=\frac{(2 n+2 \alpha+1) n ! \Gamma(n+2 \alpha+1)}{2^{2 \alpha+1}[\Gamma(n+\alpha+1)]^{2}} \int_{1}^{1} g(x)\left(1-x^{2}\right)^{\alpha} P_{n}^{(\alpha, \alpha)}(x) d x .
$$

Suppose now that $g(x)$ is the Laplace transform of some $f(t)$,

$$
g(x)=\mathscr{L}\{f(t)\}=\int_{0}^{\infty} e^{-x t} f(t) d t=\sum_{n=0}^{\infty} A_{n} P_{n}^{(\alpha, \alpha)}(x)
$$

To determine the $A_{n}$ 's replace the kernel of the Laplace transform by its Neumann series $[1$, v. 2 : p. 98 , No. (1); p. 175 , No. (16); p. 174, No. (6); and the duplication formula for the gamma function].

$$
\begin{aligned}
e^{-x t} & =\sum_{n=0}^{\infty}(-)^{n} \Omega_{n} \frac{I_{n+\alpha+1 / 2}(t)}{t^{\alpha+1 / 2}} P_{n}^{(\alpha, \alpha)}(x), \\
\Omega_{n} & =\frac{2^{1 / 2-\alpha} \pi^{1 / 2}\left(n+\alpha+\frac{1}{2}\right) \Gamma(n+2 \alpha+1)}{\Gamma(n+\alpha+1)} .
\end{aligned}
$$

Then (4) yields

$$
A_{n}=e^{(n-\alpha-1)[\pi i / 2]} \Omega_{n} \Im C\left\{\frac{f(t)}{t^{\alpha+1}}\right\}_{\substack{y=i \\ y=n+\alpha+1 / 2}},
$$

Received December 28, 1959; revised August 4, 1960. This work was supported by the United States Air Force through the Wright Air Development Division. 


$$
\operatorname{se}\{F(t)\}=\int_{0}^{-} F(t) J_{\nu}(y t)(y t)^{1 / 2} d t .
$$

$\mathfrak{S C}\{F(t)\}$ denotes the Hankel transform of $F(t)$ [2].

When $\alpha=-\frac{1}{2}$, (7) furnishes the coefficients for the Chebyshev expansion

$$
g(x)=\int_{0}^{\infty} e^{-x t} f(t) d t=\sum_{n=0}^{\infty} C_{n} T_{n}(x), \quad-1 \leqq x \leqq 1,
$$

where

$$
C_{n}=\epsilon_{n} e^{(n-1 / 2)[r i / 2)} \mathfrak{x C}\left\{\frac{f(t)}{t^{1 / 2}}\right\}_{\substack{x=i \\
n=n}}, \quad \epsilon_{n}=\begin{aligned}
& 1, n=0, \\
& 2, n>0 .
\end{aligned}
$$

If we replace $t$ by $i t$ in (5), we find that the same method is applicable when $g(x)$ is a Fourier transform of $f(t)$. We omit details, but the key results for the sine and cosine transforms are as follows.

$$
\begin{aligned}
& g_{1}(x) \\
& g_{2}(x)
\end{aligned}=\int_{0}^{\infty} f(t) \frac{\sin }{\cos }(x t) d t=\sum_{n=0}^{\infty} C_{n} C_{n}^{(a, a)}(x), \quad-1 \leqq x \leqq 1
$$

where

$$
S_{n}=\left\{\begin{array}{l}
0, \quad n \text { even, } \\
e^{(n-1)(\pi i / 2)} \Omega_{n} 3 c\left\{\frac{f(t)}{t^{a+1}}\right\}_{\substack{y=1 \\
y=n+\alpha+1 / 2}}, \quad n \text { odd },
\end{array}\right.
$$

and

$$
C_{n}=\left\{\begin{array}{l}
0, \quad n \text { odd, } \\
e^{n \pi i / 2} \Omega_{n} x \in\left\{\frac{f(t)}{t^{\alpha+1}}\right\}_{\substack{p=1 \\
n=n+\infty+1 / 2}},
\end{array}\right.
$$
Then

3. The Chebyshev Expansion for $1 /(y+a)^{k}$. Let $g(x)=\left[\frac{x+1}{2}+a\right]^{-k}$.

$$
\mathcal{L}^{-1}\{g(x)\}=\frac{2^{k}}{(k-1) !} e^{-(2 a+1) t^{k-1}}=f(t) .
$$

Use (10) and let $y=\frac{x+1}{2}$. Then $T_{n}(2 y-1)=T_{n}^{*}(y), 0 \leqq y \leqq 1$, is the shifted Chebyshev polynomial [3] and

$$
\begin{aligned}
\frac{1}{(y+a)^{k}} & =\left\{\sum_{n=0}^{\infty} \frac{\epsilon_{n}(-)^{n}(k+n-1) !}{(k-1) !} P_{k-1}^{-n}\right. \\
& \left.\cdot\left[\frac{2 a+1}{2 \sqrt{a^{2}+a}}\right] T_{n}^{*}(y)\right\} /\left(a^{2}+a\right)^{k / 2} \quad 0 \leqq y \leqq 1, \quad a>0,
\end{aligned}
$$

where $P_{r}^{\mu}(x)$ is the Legendre function $[1$, v. 1, p. 120]. For $k=1,(15)$ agrees with a result of Luke [4]. 


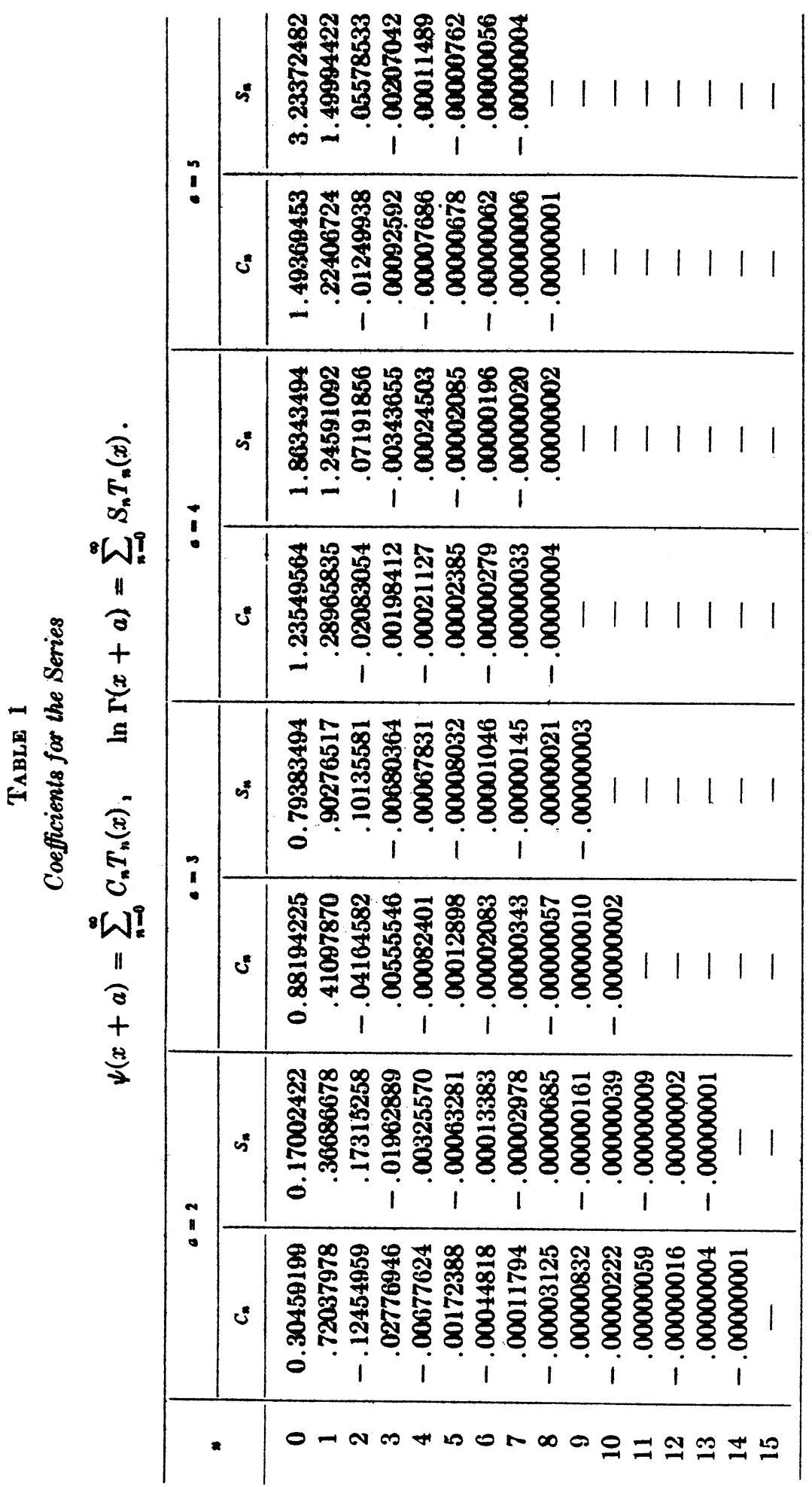


4. The Psi and Log Gamma Functions. These examples show how a property of the Laplace transform may be used to advantage when applying (4) and (8), We know that

$$
\mathscr{L}\left\{e^{-a t} f(t)\right\}=g(x+a) .
$$

If $g(x)$ cannot be expanded in symmetric Jacobi polynomials, $a$ in (16) can often be chosen so that $g(x+a)$ has a convergent expansion. Let

$$
g(x)=\psi^{(m)}(x)=D^{m+1} \log \Gamma(x) .
$$

Since $\psi^{(m)}(x)$ has poles at zero and the negative integers, we cannot expand the function over $-1 \leqq x \leqq 1$. However, if

$$
g(x)=\psi^{(m)}(x+a),
$$

then

$$
f(t)=\mathfrak{L}^{-1}\{g(x)\}=(-)^{m+1} e^{-a t} t^{m}\left[1-e^{-t}\right]^{-1},
$$

and if $\operatorname{Re}(a)>1,(7)$, and in particular (10), may be used since (18) is analytic for $|x| \leqq 1$. Substituting (19) in (10) and expanding $\left(1-e^{-t}\right)^{-1}$ by the binomial theorem, we have

$$
C_{n}=-\left.\epsilon_{n} \sum_{k=0}^{\infty} \frac{d^{m}}{d x^{m}}\left[\frac{\left(\sqrt{x^{2}-1}-x\right)^{n}}{\sqrt{x^{2}-1}}\right]\right|_{x=k+a} .
$$

Setting $m$ equal to zero, we get

\begin{tabular}{|c|c|c|c|c|}
\hline \multirow{2}{*}{$n$} & \multicolumn{2}{|c|}{$a=2$} & \multicolumn{2}{|c|}{$a=5$} \\
\hline & $A_{2 n}$ & $B_{2 n+1}$ & $A_{2 n}$ & $B_{2 n+1}$ \\
\hline $\mathbf{0}$ & 0.1352962627 & 1.6980909708 & -0.9631315550 & 20857821107 \\
\hline 1 & -.4232751922 & $\begin{array}{r}-0955849521 \\
-.0958\end{array}$ & -1.1310316550 & $\begin{array}{r}.6704259749 \\
-.67\end{array}$ \\
\hline 2 & .0182227219 & .0029578196 & $.3+66170891$ & $.15186687+2$ \\
\hline 3 & -.0004157650 & -.0000514215 & -.0569843620 & -.0186143512 \\
\hline 4 & .0000056716 & .0000005642 & .0053747844 & .0013896747 \\
\hline $\mathbf{5}$ & -.0000000511 & -.0000000042 & -.0003252237 & -.0000695137 \\
\hline 6 & .0000000003 & - & .0000136729 & .0000024908 \\
\hline 7 & - & 一 & -.0000004226 & -.0000000671 \\
\hline 8 & - & - & .0000000100 & .0000000014 \\
\hline 9 & - & - & -.0000000002 & - \\
\hline
\end{tabular}

$$
C_{n}=-\epsilon_{n} \sum_{k=0}^{\infty} \frac{\left[\sqrt{(k+a)^{2}-1}-(k+a)\right]^{n}}{\sqrt{(k+a)^{2}-1}}, \quad n \geqq 1 .
$$

TABLE 2

Coefficients for the Series

$$
\begin{array}{lrl}
C i(x)=\int_{\infty}^{x} \frac{\cos t}{t} d t=\log (x)+\sum_{n=0}^{\infty} A_{2 n} T_{2 n}\left(\frac{x}{a}\right), & 0<x \leqq a \\
S i(x)=\int_{0}^{x} \frac{\sin t}{t} d t=\sum_{n=0}^{\infty} B_{2 n+1} T_{2 n+1}\left(\frac{x}{a}\right), & & -a \leqq x \leqq a
\end{array}
$$


If $n=0,(21)$ diverges, and for $n=1$ the series is slowly convergent, but since $T_{n}(1)=1, T_{n}(-1)=(-)^{n}$, we may solve for $C_{0}$ and $C_{1}$ in terms of higher computable coefficients, i.e.,

$$
\left\{\begin{array}{l}
C_{0}=\frac{\psi(a+1)+\psi(a-1)}{2}-\sum_{k=1}^{\infty} C_{2 k}, \\
C_{1}=\frac{\psi(a+1)-\psi(a-1)}{2}-\sum_{k=1}^{\infty} C_{2 k+1} .
\end{array}\right.
$$

Integration of the series defined by (21) yields a Chebyshev expansion for ln $\Gamma(x+a)$ because [3]

$$
\int T_{n}(x) d x=\frac{1}{2}\left[\frac{T_{n+1}(x)}{n+1}-\frac{T_{n-1}(x)}{n-1}\right]+C .
$$

In Table 1 are listed coefficients for the Chebyshev expansions of $\psi(x+a)$ and $\log \Gamma(x+a), a=2(1) 5, n=0(1) 15$ to $8 \mathrm{D}$.

5. The Sine and Cosine Integrals. For examples of (11)-(13) let

$$
\begin{aligned}
& \begin{array}{l}
g_{1}(x)=\begin{array}{l}
(1-\cos a x) / x \\
g_{2}(x)
\end{array}=\int_{0}^{\infty} f(t) \frac{\sin x t}{\cos x t} d t,
\end{array} \\
& f(t)= \begin{cases}1, & 0<x<a, \\
0, & a<x<\infty .\end{cases}
\end{aligned}
$$

Using [2, v. 2, p. 333, No. (1)] to evaluate (12) and (13) for $\alpha=-\frac{1}{2}$, we find that

$$
\begin{aligned}
& S_{n}= \begin{cases}0, & n \text { even, } \\
4 e^{(n-1)[r i / 2]} \sum_{k=0}^{\infty} J_{n+2 k+1}(a), & n \text { odd, }\end{cases} \\
& C_{n}= \begin{cases}0, \quad n \text { odd }, \\
2 \epsilon_{m} e^{n \pi i / 2} \sum_{k=0}^{\infty} J_{n+2 k+1}(a), \quad n \text { even. }\end{cases}
\end{aligned}
$$

Let $a=2$ and $j$ in (26) and (27), and use [1, v. 2, p. 145, No. (6)] and (23) to obtain the expansion whose coefficients are listed in Table 2.

The author wishes to thank Yudell L. Luke and also Charles Himmelberg and Jerry Fields for their helpful suggestions during the preparation of this paper.

Midwest Research Institute

Kansas City, Missouri

1. A. Erdelyi, W. Magnus, F. Oberagtringer \& F. G. Tricom, Higher Transcendental Functions, Vol. 1 and 2, McGraw-Hill, New York, 1953.

2. A. Erdezyi, W. Magnus, F. Oberhetringer \& F. G. Triconi, Tables of Integral Transfornis, Vol. 1 and 2, MeGraw-Hill, New York, 1953.

3. C. LAvczos, "Tables of Chobyshev polynomials, $S_{n}(x)$ and $C_{n}(x)$," Nat. Bur. Standards, A ppl. Math. Ser. No. 9, U. S. Government Printing Office, Washington, D. C., 1952.

4. Y. L. LUKE, "On the computation of $\log Z$ and $\arctan Z$," MTAC, v, 11, 1957, p. 16-18. 